\title{
Primary Angioplasty: Efficacy and Outcomes
}

\author{
lan Patrick Kay and Brittany Georgia Kay
}

\subsection{Introduction}

Internationally ischaemic heart disease (IHD) is the single most common cause of death, and its frequency is increasing. The relative incidences of ST-elevation myocardial infarction (STEMI) and non-ST-elevation myocardial infarction (NSTEMI) are decreasing and increasing, respectively. In European countries, the incidence rate for STEMI ranges from 43 to 144 per 100,000 population per year. Similarly, the reported adjusted incidence rates from the USA decreased from 133 per 100,000 in 1999 to 50 per 100,000 in 2008, whereas the incidence of NSTEMI remained constant or increased slightly. STEMI is more common in younger than in older people and is more common in men than in women.

Mortality in STEMI is associated with advanced age, Killip class, time delay to treatment, presence of emergency medical system (EMS)-based STEMI networks, treatment strategy, history of MI, diabetes mellitus, renal failure, number of diseased coronary arteries and left ventricular ejection fraction (LVEF). Overall mortality from STEMI remains substantial with the in-hospital mortality of unselected patients with STEMI in the national registries of the European Society of Cardiology (ESC) member countries varying between $4 \%$ and $12 \%$. Whilst reported 1-year mortality among STEMI patients in other registries is approximately $10 \%$.

Although IHD develops later in women compared with men, MI remains a leading cause of death in women. Acute coronary syndrome (ACS) occurs three to four times more often in men than in women below the age of 60 years, but after the age of 75, women represent the majority of patients. Women may present with atypical symptoms - seen in up to $30 \%$ in some registries. It is therefore important to be

\author{
I. P. Kay $(\bowtie)$ \\ Mercy Angiography, Auckland, New Zealand \\ B. G. Kay \\ University of Tasmania, Hobart, TAS, Australia
}


vigilant when encountering a potential ACS presentation in a female. Women also have a higher bleeding risk peri- and post-PCI. Several studies have suggested a poorer outcome in females suffering MI, related to older age and more comorbidities. Some studies have indicated that women may undergo fewer interventions than men and receive reperfusion therapy less frequently. The guidelines stress the fact that women and men receive equal benefit from a reperfusion strategy and STEMIrelated therapy and that both genders must be managed in a similar fashion.

\subsection{Treatment of STEMI}

Patients with chest pain suggestive of an ACS and having ECG evidence of an acute STEMI are candidates for reperfusion therapy with either primary percutaneous coronary intervention (PPCI) or fibrinolytic therapy. Patients with typical symptoms in the presence of a new left or right bundle branch block or a true posterior STEMI are also considered eligible.

Coronary reperfusion with PPCI or fibrinolytic therapy improves outcomes in patients with acute ST-elevation MI. However, PPCI is the reperfusion therapy of choice compared to fibrinolysis because it achieves a higher rate of TIMI 3 flow (more than 90\%), does not carry the risk of intracranial haemorrhage and is associated with improved outcomes. The time to onset of reperfusion therapy is a critical determinant of outcome with both PPCI and fibrinolysis.

Definitions - The delay in treatment delivery between the onset of symptoms and primary percutaneous coronary intervention (PPCI) is termed the treatment delay and has the following components:

- Patient delay-This is the time between onset of symptoms and the call to the emergency medical system (EMS).

- Prehospital system delay-This is the time between the EMS call and the arrival at the PCI centre and is equivalent to the time of EMS evaluation, prehospital electrocardiogram obtainment (if any) and transportation to the hospital. For patients transferred from a local hospital to a PCI centre, this is sum of the time between the EMS call and arrival at the local hospital, the time at the local hospital and the time between hospitals (second transfer time).

- Door-to-balloon delay (D2B) - This is the time between arrival at the PCI centre and PPCI. Time of PPCI refers to the time of coronary intervention with a balloon, stent or other intervention, not to be confused with the time of arrival in the catheterization laboratory or the time of vascular access.

- First medical contact (FMC) - This concept is upgraded in the 2017 version of the ESC guidelines. This is the time point when the patient is initially assessed by a physician, paramedic or nurse who can obtain and interpret an ECG. This can be prehospital or in-hospital. Note if FMC is in the community and if the patient is transferred directly to the catheter laboratory, then the concept of door to balloon is dropped.

- System delay-This is the sum of the prehospital system and door-to-balloon delays. 
In this schema, patient delay is not easily corrected by the healthcare system, while strategies to shorten other delays are potentially modifiable. Such an effort will be useful if longer time intervals are associated with worse outcomes.

\subsection{Fibrinolytic Therapy}

Fibrinolytics are most effective when given within the first $2 \mathrm{~h}$ after the onset of symptoms. However, many patients with STEMI present late after the development of symptoms. In different registries of patients, the time from symptom onset to hospital presentation was $\geq 4 \mathrm{~h}$ in $50 \%$, more than $6 \mathrm{~h}$ in $40 \%$ and more than $12 \mathrm{~h}$ in $9-31 \%$. Delay is greatest in women, older adults, low socioeconomic and ethnic minority groups and those with symptoms that occur between 6 P.M. and 6 A.M.

\subsection{Late Presentation PPCI}

In contrast to fibrinolysis, revascularization after $12 \mathrm{~h}$ with PCI may still be beneficial in the $9-31 \%$ of patients with STEMI who present more than $12 \mathrm{~h}$ after the onset of symptoms. The randomized trials that have evaluated late PCI have included patients at different time periods after symptom onset, ranging from $12 \mathrm{~h}$ to up to 28 days. Some have demonstrated an improvement in left ventricular function with PCI, but none have demonstrated a significant benefit on death.

The American College of Cardiology/American Heart Association (ACC/AHA) guidelines suggest that it is reasonable to perform PPCI for patients with onset of symptoms within the prior $12-24 \mathrm{~h}$ who have one or more of the following:

- Severe heart failure

- Haemodynamic or electrical instability

- Persistent ischaemic symptoms

In contrast, the task force did not recommend PPCI in stable, asymptomatic patients presenting more than $12 \mathrm{~h}$ after symptom onset.

\subsection{Door-To-Balloon Time (D2B)}

D2B is well studied and is predictive of in-hospital mortality. Longer D2B times result in poorer outcomes as evidenced by both observational studies and randomized trials:

- The NRMI-3 and NRMI-4 registries (1999-2002) reported 29,222 ST-elevation myocardial infarction (STEMI) patients who were treated with PCI within $6 \mathrm{~h}$ of presentation. Longer D2B times were significantly associated with increased inhospital mortality $(3.0 \%, 4.2 \%, 5.7 \%$ and $7.4 \%$ for $\mathrm{D} 2 \mathrm{~B}$ time of $\leq 90 \mathrm{~min}$, 
91-120 min, 121-150 min and >150 min, respectively). Patients with D2B times $>90$ min had a significant increase in mortality compared to those with D2B times $\leq 90 \mathrm{~min}$ (odds ratio 1.42).

- The CADILLAC and HORIZONS-AMI trials analysed 4548 patients; short D2B times $(\leq 90 \mathrm{~min})$ were associated with a significantly lower mortality rate at 1 year compared to longer times $(3.1 \%$ vs. $4.3 \%$; hazard ratio $0.72,95 \% \mathrm{CI}$ 0.52-0.99).

Despite the evidence that longer D2B times lead to worse outcomes, improvement in D2B times has not lead to improvement in survival rates. In a study of nearly 100,000 STEMI patients in the US CathPCI Registry of the National Cardiovascular Data Registry who underwent primary PCI between July 2005 and June 2009, median D2B times fell from 83 to $67 \mathrm{~min}$, comparing the first to the last 12 months of the time period. Despite this significant improvement, there was no change in risk-adjusted in-hospital mortality $(5.0 \%$ vs. $4.7 \%$, respectively; $p=0.34)$.

Although there is a direct relationship between increasing mortality and longer D2B time, it may be difficult to demonstrate further improvements in survival with shorter D2B times, particularly if they are in the order of magnitude of minutes. This is in part related to the impact of total ischaemic time on mortality.

Outcomes in patient subgroups-The issue of whether the improvement in survival with short D2B times is delivered equally to all patients has been studied, and two subgroups of patients appear to benefit the most: those who present early after symptom onset and those at high risk.

Early versus late presentation-With regard to early versus late presentation, it appears that short D2B time is associated with improved outcome in early presenters more than in late presenters:

- In a study of 2322 patients who underwent PPCI comparing short versus long D2B time ( $<2$ vs. $>2 \mathrm{~h}$ ), there was a significant decrease in 7-year mortality in patients presenting $\leq 3$ hours from symptom onset (15.0\% vs. $24.7 \%$ ), but not in patients presenting after $3 \mathrm{~h}(18.5 \%$ vs. $21.1 \%)$.

- Similarly in 4548 patients who underwent PPCI win the CADILLAC and HORIZONS-AMI trials, short ( $\leq 90 \mathrm{~min})$ compared to long D2B times were associated with a significantly lower 1-year mortality rate in patients with early presentation ( $\leq 90 \mathrm{~min}$ vs. $\geq 90 \mathrm{~min}$ ), but not those with later presentation $(1.9 \%$ vs. $3.8 \%, \mathrm{HR}=0.86,95 \% \mathrm{CI} 0.26-0.93$ and $4.0 \%$ vs. $4.6 \%, \mathrm{HR}=0.86,95 \% \mathrm{CI}$ $0.58-1.28$, respectively).

Patient risk category-High-risk patients (defined as one or more of Killip class 3 or 4 , heart failure, age $>70$ years or anterior MI) appear to benefit more from short D2B time:

- In the study of 2322 patients discussed above, there was a significant decrease in 7-year mortality with shorter D2B times ( $<2$ vs. $\geq 2 \mathrm{~h})$ in high-risk $(21.5 \%$ vs. $32.5 \%)$ but not low-risk patients $(9.2 \%$ vs. $10.8 \%)$. 
- In the CADILLAC and HORIZONS-AMI trials, short compared to long D2B times showed a trend toward lower 1-year mortality in both high- and low-risk groups $(5.7 \%$ vs. $7.4 \%$ and $1.1 \%$ vs. $1.6 \%$, respectively). In patients presenting early ( $\leq 90 \mathrm{~min}$ ), the hazard ratios for mortality rate in patients with short versus long D2B times were identical for high- versus low-risk patients, but the absolute mortality rate differences were greater in high-risk patients $(3.3 \%$ vs. $0.7 \%)$. In patients presenting late (>90 min), mortality was similar with short and long D2B times in both high- and low-risk patients.

The findings above suggest that short D2B times are critically important in patients who present early, especially in high-risk patients.

\subsection{Time from First Medical Contact with the Healthcare System (System Delay)}

System delay is the sum of the prehospital system and door-to-balloon delays. For patients first brought to a hospital without PCI capability, the system delay has multiple components.

The associations between healthcare delays and mortality were evaluated in a study of 6209 patients with STEMI from 2002 to 2006 by Danish researchers. Approximately one-third of these patients were triaged in the field to a PCI centre, and two-thirds were admitted to a local hospital and then transferred to a PCI centre. The median follow-up time was 3.4 years, and the cumulative 1-year mortality was $9.3 \%$. The following findings were noted:

- In the univariate analysis of the components of delay (system, prehospital system, door-to-balloon, treatment and patient), system delay had the strongest association with mortality (hazard ratios [HR] 1.22, 1.19, 1.13, 1.054 and 1.042, respectively). All HR were significant.

- For system delays of 0-60 min, 61-120 min, 121-180 min and 181-360 min, the long-term cumulative mortality was $15.4 \%, 23.3 \%, 28.1 \%$ and $30.8 \%$, respectively.

- In the multivariate analysis, treatment delay and patient delay were not associated with mortality, but system delay was independently associated with mortality (HR 1.10, 95\% CI 1.01-1.16 per 1-hour delay). The main components of system delay, prehospital system delay and door-to-balloon delay were similarly associated with mortality (HR $1.10,95 \%$ CI $1.02-1.18$ and 1.14 , 95\% CI $1.05-$ 1.24 , respectively).

This study demonstrates the negative impact of increasing system delay on mortality. It does not allow any firm conclusion regarding the time at which using a fibrinolytic agent would be preferable. Therefore, the earlier a patient presents (and thus a higher baseline risk), the less D2B delay that is acceptable. Thus, fibrinolytic therapy is an important option for patients who present within the first $3 \mathrm{~h}$ and who are at a low risk of bleeding. 


\subsection{Direct Transfer from the Community}

Mortality is improved with direct transfer from the community to a PCI hospital compared with evaluation and treatment at a closer hospital without PCI capability. The feasibility and impact of this strategy on D2B delay were evaluated in a nonrandomized comparison of 344 patients with chest pain of less than $12 \mathrm{~h}$ duration and ST-segment elevation characteristic of acute MI in 2005 and 2006. In this study, 135 patients were referred directly from the community by paramedics trained in ECG interpretation, and 209 patients were referred directly from regional emergency departments. The median D2B time, defined as the time between arrival at the first hospital to first balloon inflation, was significantly shorter in patients referred from the community (69 vs. $123 \mathrm{~min}$ ), and the percent of patients with D2B times of less than 90 min was significantly higher ( 80 vs. 12).

For patients with STEMI diagnosed with a prehospital ECG who are transported to a PCI-capable hospital, one way to shorten the time to reperfusion is to bypass the emergency department (ED) and take the patient directly to the catheterization laboratory. The efficacy of this approach was addressed in a study of 12,158 STEMI patients, $10.5 \%$ of whom bypassed the ED. The time from first medical contact (FMC) to device activation was shorter in these individuals compared to those who went to the ED (68 vs. $88 \mathrm{~min} ; p<0.0001)$. There was a trend toward lower mortality $(2.7 \%$ vs. $4.1 \%$; adjusted odds ratio $0.69,95 \%$ CI $0.45-1.03)$.

Therefore, the optimal strategy for STEMI patients diagnosed with a prehospital ECG should be to transfer the patient directly to the catheterization laboratory of a PCI-capable hospital (bypassing the ED) if the following two criteria are met:

1. The patient is haemodynamically stable.

2. The patient is received by personnel (including one physician) qualified to care for critically ill patients.

Transfer from a non-PCI centre-The poor outcomes seen in patients who are initially evaluated at hospitals without on-site PCI capability are of concern. The time spent at the first hospital, the subsequent transfer time and the time spent at the receiving hospital prior to arrival in the catheterization laboratory are sources of longer D2B. The frequency, magnitude and clinical impact of delays occurring at each of these time intervals were examined in a prospective study of 2034 patients referred to a PCI centre for primary PCI. The following findings were noted:

- Delays occurred most frequently in the ED at the referral hospital, with less delay seen at the PCI centre or in transport (64\%, 16\% and 13\%, respectively).

- For the referral hospital, the most frequent reasons for delay were awaiting transport and ED delays (26\% and 14\%, respectively).

Interestingly, diagnostic difficulties, including non-diagnostic initial ECGs, led to the greatest delay, but these had limited or no impact on mortality. 


\subsection{The Door-In to Door-Out (DIDO) Time}

This is defined as the duration of time from arrival to discharge at the first (non-PCI capable) hospital. The DIDO time is probably modifiable, as opposed to the nonmodifiable delay caused by an additional ambulance ride. The optimum DIDO measure is $<30 \mathrm{~min}$, (AHA/ACC 2008). The relationship between DIDO time and outcomes was evaluated in in the Acute Coronary Treatment and Intervention Outcomes Network (ACTION) Registry-Get With the Guidelines (GWTG) between 2007 and 2010. The following results were reported:

- The median DIDO time was 68 min, with only $11 \%$ of patients having a goal DIDO time of less than 30 min.

- Predictors of longer DIDO times included older age, female sex, off-hours presentation and non-emergency medical services presentation to the first hospital.

- Patients with DIDO times less than 30 min had significantly shorter door-toballoon times ( 85 vs. $127 \mathrm{~min}$ ) and a lower in-hospital mortality rate (2.7\% vs. $5.9 \%)$.

AHA/ACC and ESC Recommendations - The 2013 American College of Cardiology Foundation/American Heart Association guideline for the management of ST-elevation myocardial infarction (STEMI) recommends the timely performance of primary percutaneous coronary intervention (PCI) and sets the following time goals:

- For patients who initially arrive at or are transported to a non-PCI-capable hospital, the first medical contact to device time should be 120 min or less.

- For patients who initially arrive at or are transported to a PCI-capable hospital, the first medical contact to device time should be 90 min or less.

- The 2012 European Society of Cardiology guideline on STEMI recommended shorter time intervals of 90 and $60 \mathrm{~min}$, respectively.

\begin{tabular}{l|l}
\hline ESC 2017 Summary of important time targets & $\begin{array}{l}\text { Time } \\
\text { targets }\end{array}$ \\
\hline Maximum time from FMC to ECG and diagnosis & $\leq 10 \mathrm{~min}$ \\
\hline $\begin{array}{l}\text { Maximum time delay from STEMI diagnosis to PPCI (wire crossing) to choose } \\
\text { PPCI strategy over fibrinolysis (if this target cannot be met consider fibrinolysis) }\end{array}$ & $\leq 120 \mathrm{~min}$ \\
\hline $\begin{array}{l}\text { Maximum time from STEMI diagnosis to wire crossing in patients presenting to } \\
\text { PPCI hospitals }\end{array}$ & $\leq 60 \mathrm{~min}$ \\
\hline Maximum time from STEMI diagnosis to wire crossing in transferred patients & $\leq 90 \mathrm{~min}$ \\
\hline $\begin{array}{l}\text { Maximum time from STEMI diagnosis to bolus or infusion of fibrinolysis in } \\
\text { patients unable to meet PPCI target times }\end{array}$ & $\leq 10 \mathrm{~min}$ \\
\hline Time delay from start of fibrinolysis to evaluation of its efficacy & $60-90 \mathrm{~min}$ \\
\hline Time delay from start of fibrinolysis to angiography (if fibrinolysis successful) & $2-24 \mathrm{~h}$ \\
\hline
\end{tabular}

Ref: academic.oup.com/eurheartj/advance-article/doi/10.1093/eurheartj/ehx393/4095042 


\subsection{Non-system Factors Leading to Delay}

In a study of nearly 83,000 patients with STEMI in the US PCI Registry (20092011), non-system delays occurred in $14.7 \%$. Typical reasons cited included delays in gaining consent, difficult vascular access and difficulty in crossing the lesion. As expected, and consistent with an increase in D2B time, the in-hospital mortality was greater in those with compared to those without non-system delay (15.1\% vs. $2.5 \%$; $p<0.01$ ) even after PCI.

Prognosis after Primary PCI-In a registry study of 2804 patients, 30-day, 1-year and 5-year all-cause (and cardiac) mortality rates were 7.9 (7.3)\%, 11.4 (8.4)\% and $23.3(13.8) \%$, respectively. In this study, the main causes of cardiac death within the first 30 days were cardiogenic shock and anoxic brain injury. After 30 days, causes of death were predominantly noncardiac, with malignancies and pulmonary diseases dominating.

Other risk factors are discussed below:

TIMI flow grade - Patients with normal blood flow in the infarcted artery at the end of the procedure have a better prognosis than those who do not.

Electrocardiographic markers-Electrocardiographic markers can successfully predict outcome in the broad range of patients with ST-elevation myocardial infarction. The prognostic utility of six different methods for evaluating the extent of ST-segment elevation resolution after PCI was assessed in 4866 patients enrolled in the APEX-AMI trial. All six methods were successful in predicting outcomes of death or the composite of death, cardiogenic shock or heart failure at 90 days. One of these, the measurement of the residual, absolute ST elevation in the single, most affected (worst) lead $30 \mathrm{~min}$ after PCI, performed as well as the more complex methods.

Infarct size - Larger infarct size after PPCI is associated with worse outcomes. In a patient-level meta-analysis of ten randomized trials of primary PCI in which infarct size was assessed with cardiac magnetic resonance imaging or technetium$99 \mathrm{~m}$ sestaMIBI single-photon emission computed tomography within 1 month of STEMI, the following was noted:

- Median infarct size (percent of left ventricular myocardial mass) was $17.9 \%$.

- One-year estimates of all-cause mortality, re-infarction and heart failure hospitalization were $2.2 \%, 2.5 \%$ and $2.6 \%$, respectively.

- There was a strong, graded response between infarct size and mortality and hospitalization for heart failure at 1 year.

Other risk factors - Other risk factors including Killip class, age and the number of diseased vessels are also important. These parameters have been incorporated into three risk models that have been prospectively validated: the Zwolle risk index, the TIMI risk score and the CADILLAC risk score. 


\subsection{What Is New in the ESC 2017 Guidelines on AMI-STEMI? New/Revised Concepts}

\section{Strategy Selection and Time Delays:}

- Clear definition of first medical contact (FMC).

- Definition of "time 0" to choose reperfusion strategy (i.e. the strategy clock starts at the time of "STEMI diagnosis").

- Selection of PCI over fibrinolysis: when anticipated delay from "STEMI diagnosis" to wire crossing is $\leq 120 \mathrm{~min}$.

- Maximum delay time from "STEMI diagnosis" to bolus of fibrinolysis agent is set in 10 min.

- "Door-to-balloon" term eliminated from guidelines.

- See www.escardio.org/guidelines 2017 ESC Guidelines for the Management of AMI-STEMI (European Heart Journal 2017—doi:10.1093/eurheartj/ ehx(095).

\subsection{Summary}

The following conclusions can be reached from the clinical trials and observational studies discussed above:

- Primary percutaneous coronary intervention (PPCI) with stenting, if performed in a timely manner, is associated with better outcomes than fibrinolysis.

- The time from symptom onset to PCI has not been shown to be an important determinant of outcome. At the least, the benefit from PPCI is less dependent upon the time from symptom onset than is fibrinolysis.

- The time from hospital arrival to PCI (D2B) is an important determinant of benefit, with the best outcomes occurring when the time to PCI is $90 \mathrm{~min}$ or less. This measure is likely to be replaced with the greater use of FMC.

- Increasing system delay is associated with worse outcomes.

- Patients who are transferred to a PCI centre have better outcomes than those treated with fibrinolysis at the presenting hospital if PPCI is delivered according to guideline standards. Destination protocols for emergency medical system that bypass non-PPCI-capable hospitals and thereby shorten system delays to PPCI have been associated with improved outcomes in ST-elevation myocardial infarction.

- Hospitals should adapt strategies to reduce door-to-balloon times and thereby improve outcomes in STEMI patients treated with PPCI.

- Late PCI to open an occluded artery should be considered in patients with severe heart failure, hemodynamic or electrical instability or persistent ischemic symptoms. 
- PPCI should not be performed in hospitals without on-site cardiac surgery unless they meet specific criteria, including having a proven plan for rapid transport to a cardiac surgery operating room in a nearby hospital and having appropriate haemodynamic support capability for transfer.

\section{Conclusion}

The acute ischaemia caused by SCAD in the left circumflex artery in this case acts as a physical and emotional stressor resulting in TC. Hence postischaemic myocardial stunning from SCAD may induce TC, and SCAD-induced MI may trigger rather than exclude TC.

\section{Further Readings}

Lambert L, Brown K, Segal E, et al. Association between timeliness of reperfusion therapy and clinical outcomes in ST-elevation myocardial infarction. JAMA. 2010;303:2148.

Terkelsen CJ, Sørensen JT, Maeng M, et al. System delay and mortality among patients with STEMI treated with primary percutaneous coronary intervention. JAMA. 2010;304:763.

www.escardio.org/guidelines 2017 ESC Guidelines for the Management of AMI-STEMI

(European Heart Journal 2017. doi:https://doi.org/10.1093/eurheartj/ehx095)

Open Access This chapter is licensed under the terms of the Creative Commons Attribution 4.0 International License (http://creativecommons.org/licenses/by/4.0/), which permits use, sharing, adaptation, distribution and reproduction in any medium or format, as long as you give appropriate credit to the original author(s) and the source, provide a link to the Creative Commons license and indicate if changes were made.

The images or other third party material in this chapter are included in the chapter's Creative Commons license, unless indicated otherwise in a credit line to the material. If material is not included in the chapter's Creative Commons license and your intended use is not permitted by statutory regulation or exceeds the permitted use, you will need to obtain permission directly from the copyright holder.

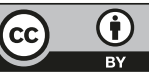

